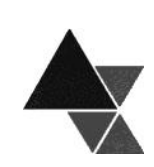

\title{
Determinantes da insegurança alimentar no Brasil em 2004 e 2009
}

\author{
Rodolfo Hoffmann ${ }^{1}$
}

Os dados sobre insegurança alimentar nos domicílios brasileiros coletados na Pesquisa Nacional por Amostra de Domicílios (PNAD) em 2004 e 2009 são analisados com o objetivo de avaliar quais são as características de cada domićlio que afetam a probabilidade de ele apresentar insegurança alimentar moderada ou grave. Confirma-se a forte relação negativa entre nível da renda domiciliar per capita e a insegurança alimentar. Como entre 2004 e 2009 ocorreu substancial crescimento da renda e redução da desigualdade, com crescimento mais intenso da renda dos relativamente pobres, poderia se prever a queda na incidência de insegurança alimentar. Isso efetivamente ocorre para a insegurança alimentar moderada (cuja incidência cai de 12,3\% para 6,5\% dos domicílios) e para a insegurança alimentar grave (cuja incidência cai de 6,3\% para 4,9\% dos domićlíos). Estranhamente, a incidência de insegurança alimentar leve aumenta de 16,1\% em 2004 para 18,7\% em 2009. O modelo de lógite estimado mostra que a redução na incidência de insegurança alimentar moderada ou grave foi maior do que o esperado com base no conjunto de variáveis explanatórias considerado. Quando se considera apenas a insegurança alimentar grave ocorre o inverso: a redução foi menor do que a esperada. Esses resultados são discutidos tendo em vista o caráter parcialmente subjetivo da escala usada para medir o grau de insegurança alimentar.

Palavras-chave: segurança alimentar, modelos de lógite, Brasil.

\section{Determinants of Food Insecurity in Brazil in 2004 and 2009}

In 2004 and 2009 the Brazilian National Household Sample Survey (PNAD) included a complementary research on food security. These data are analyzed in order to determine which household characteristics affect the probability of it having moderate or severe food insecurity. The results confirm the strong negative relation between the level of household per capita income and the probability of food insecurity. From 2004 to 2009 income increased substantially, especially for the poor, in parallel to the decrease in income inequality. Therefore, a reduction in food insecurity was expected. That actually occurred for moderate food insecurity (which fell from $12.3 \%$ to $6.5 \%$ of the households) and for severe food insecurity (which fell from $6.3 \%$ to $4.9 \%$ of the households). Strangely, the incidence of low food insecurity increased from 16,1\% in 2004 to 18,7\% in 2009. An estimated logit model shows that the reduction in moderate or severe food insecurity was greater than the expected reduction due to changes in all explanatory variables included. However, when considering only severe food insecurity the opposite occurs: the observed reduction is lower than the one expected. These results are discussed taking into account the subjectivity of the scale used to measure food insecurity.

Key-words: food security, logit models, Brazil.

\footnotetext{
${ }^{1}$ Prof. Sênior da Escola Superior de Agricultura “Luiz de Queiroz” (ESALQ), Universidade de São Paulo (USP), com apoio do CNPq. O autor agradece a Josimar Gonçalves de Jesus e Henrique Coelho Kawamura pela leitura crítica de uma versão preliminar do artigo. Correspondência: ESALQ-LES, Cx. Postal 9. CEP 13.418900. Piracicaba-SP.E-mail: hoffmannr@usp.br.
} 


\section{INTRODUÇÃO}

O objetivo deste artigo é analisar a insegurança alimentar no Brasil utilizando os dados da Pesquisa Nacional por Amostra de Domicílios (PNAD) de 2004 e 2009, com base na Escala Brasileira de Insegurança Alimentar (EBIA) [1]. Trata-se de uma continuação (ou complementação) de artigo publicado nesta revista em 2008, analisando os dados da PNAD de 2004 [2].

Neste artigo adota-se o conceito de Segurança Alimentar subjacente à EBIA. Um domicílio tem Segurança Alimentar se seus membros têm acesso regular e permanente a alimentos suficientes. Trata-se de conceito distinto do de Segurança Alimentar e Nutricional (SAN) da Lei Orgânica de Segurança Alimentar e Nutricional de 2006.

\section{A LITERATURA SOBRE INSEGURANÇA ALIMENTAR}

$\mathrm{Na}$ literatura brasileira sobre SAN há muitos trabalhos dedicados a relatar a origem do conceito e das normas, declarações e leis sobre o tema, como, por exemplo, os dois primeiros capítulos do livro de Rocha et al. publicado em 2013 [3]. Ver também Maluf et al. [4], Instituto de Pesquisa Econômica Aplicada (IPEA) [5], Belik [6], Takagi [7], Conselho Nacional de Segurança Alimentar (CONSEA) ${ }^{[8]}$, Burity et al. ${ }^{[0]}$ e Almeida Filho e Ramos ${ }^{[10]}$

No capítulo 4 do livro de Rocha et al. mencionado no parágrafo anterior [11] (p. 94-95), lê-se que:

Por se tratar de um direito humano, como pauta a Lei Orgânica de Segurança Alimentar e Nutricional (LOSAN), todos os indivíduos de forma indiscriminada devem ser contemplados por uma política de SAN e ter acesso a uma alimentação de qualidade e adequada às suas necessidades específicas, de acordo com a fase do ciclo de vida (infância, adolescência, vida adulta, gestantes, idosos), as condições de saúdedoença, as condições socioeconômicas, a cultura, o gênero e a etnia. Uma política de SAN é, portanto, universal e, assim, impõe a superação das desigualdades de acesso dos grupos populacionais à alimentação adequada por meio de ações de governo. Para que a universalidade seja garantida, as ações de SAN devem atender as especificidades dos diferentes segmentos da população. Isso implica superar qualquer discriminação étnica, de gênero, social, de renda e cultura no acesso à alimentação adequada, ou seja, implica garantir a equidade.

Pura utopia. Conforme os objetivos do autor, esse discurso pode ser útil como bandeira política. Mas não deve ser confundido com análise científica. Nem há, no texto, tentativa de identificar as forças sociais que permitiriam atingir o desiderato de SAN que superasse qualquer "discriminação de renda".

Cabe à ciência esclarecer fatos e relações que ajudem a fazer boas leis. Mas leis e suas justificativas não podem ser confundidas com "verdades" científicas. As leis devem ser analisadas criticamente.

Como exemplo de pesquisa analítica do tema, cabe lembrar o livro já clássico de Amartya Sen sobre Famines [12] (mortandade por fome) no qual ele analisa cuidadosamente várias dessas tragédias, mostrando que havia disponibilidade de alimentos no país ou na região, mas que as pessoas não tinham entitlement (acesso legal) aos alimentos.

Em sua tese de doutorado, Gubert [13] desenvolve, com base nos dados da PNAD de 2004, um modelo para estimar a incidência de insegurança alimentar grave nos municípios brasileiros, utilizando os dados do Censo Demográfico de 20002. O passo seguinte é discutir se o método pode ser útil para que os benefícios do Programa Bolsa Família sejam mais bem focalizados.

Santos et al. [14] analisam a insegurança alimentar em Pelotas (RS). Um resultado relevante é a constatação de que nada menos do que $59 \%$ das mulheres adultas de famílias com insegurança alimentar tinham excesso de peso.

Panigassi et al. [15] tratam da "Insegurança alimentar como indicador de iniquidade". Para a ciência é melhor que os termos e as expressões tenham, na medida do possível, um sentido específico. Os autores fazem uma tentativa retórica de "enriquecer" o conceito de segurança alimentar, afirmando que é um indicador de iniquidade.

Combatendo as confusões conceituais,
Monteiro $[16,17,18]$ procura distinguir claramente a

${ }^{2}$ Há erros em expressões matemáticas e na sua interpretação nas p. 17 e 40 da tese. Como a renda é uma variável importante, seria aconselhável considerar um número maior de estratos de Renda Domiciliar Per Capita (foram considerados apenas 4). 
dimensão da fome, da desnutrição e da pobreza no Brasil.

Analisando dados da Indonésia, Gans [19] compara a eficiência econômica de transferências de renda para os pobres e subsídios aos preços de alimentos básicos como políticas alternativas de promoção da segurança alimentar. Tendo em vista a dificuldade de restringir o fornecimento de alimentos mais baratos aos que sofrem de insegurança alimentar, as transferências de renda se mostram relativamente eficientes. $\mathrm{Na}$ medida em que os parâmetros econômicos sejam semelhantes no Brasil, o resultado fortalece a opinião de que o Programa Bolsa Família é uma maneira eficiente de contribuir para promover a segurança alimentar no Brasil.

\section{METODOLOGIA}

Cientificamente, é conveniente distinguir os seguintes conceitos: a "segurança alimentar" (food security), no sentido de acesso a alimentos; a "segurança dos alimentos" (food safety), que trata da qualidade dos alimentos disponíveis; e o "estado nutricional das pessoas", que depende da segurança alimentar e da segurança dos alimentos, mas envolve também a saúde de quem ingere os alimentos.

Agregar tudo em um único conceito é analiticamente inconveniente. A EBIA [1,20], da mesma maneira que a escala americana da qual se originou [21], procura captar a percepção de insegurança alimentar por meio de

um gradiente de severidade, iniciando pelo receio da pessoa de que a família venha a sofrer privação alimentar no futuro próximo, passando pelo comprometimento da qualidade da dieta e pela limitação da quantidade de alimentos consumidos no domicílio, chegando até o nível mais grave da insegurança alimentar, que é fome entre adultos e/ou crianças da família (2006, p. 66) [20].

Aqui serão analisados os dados das PNADs de 2004 e 2009, as únicas em que foi aplicado o suplemento de segurança alimentar, com base na EBIA.

Todas as análises estatísticas apresentadas neste trabalho são ponderadas, usando os fatores de expansão da amostra da PNAD de cada ano divulgados pelo Instituto Brasileiro de Geografia e Estatística
(IBGE) após a apuração dos dados do Censo Demográfico de 2010.

Os valores monetários de 2004 foram colocados na unidade monetária usada nos dados da PNAD de 2009, usando como deflator o Índice Nacional de Preços ao Consumidor (INPC) ${ }^{3}$.

Na amostra da PNAD de 2004 há 112.530 domicílios particulares permanentes, representando uma população de 51.600.819 domicílios. Na PNAD de 2009 esses números são 120.910 e 58.566.292, respectivamente.

Para fazer as análises apresentadas adiante, foi necessário eliminar da amostra os domicílios sem declaração do valor de qualquer das variáveis a serem utilizadas (a medida de insegurança alimentar, a renda domiciliar, a cor, a idade e a escolaridade da pessoa de referência do domicilio, etc.). Também foram excluídos os domicílios com pessoa de referência indígena e os com renda domiciliar nula. Passou-se a utilizar, então, a amostra cuja dimensão está descrita na Tabela 1. Para algumas análises as duas amostras são "empilhadas", formando uma amostra agregada com 223.259 observações.

Cada domicilio é classificado em 4 categorias (com segurança alimentar, com insegurança leve, com insegurança moderada ou com insegurança grave) conforme o número de respostas afirmativas a uma lista de 15 perguntas que pode ser encontrada nas publicações do IBGE [22,23] ou no nosso trabalho anterior [2].

A escala procura captar desde situações em que há preocupação de que vai faltar comida no domicílio, até situações de fome (um morador ficar um dia inteiro sem comer, porque não havia dinheiro para comprar comida).

\footnotetext{
${ }^{3}$ Como o mês de referência das PNADs é setembro e os salários são, em geral, pagos no início do mês seguinte, foram utilizadas as médias geométricas do INPC em setembro e outubro de cada ano. Dessa maneira, considera-se que todos os valores monetários estão expressos em reais de setembrooutubro de 2009.
} 
Tabela 1. Número de domicílios na amostra e correspondente número de domicílios e pessoas na população em cada ano, após depuração da amostra

\begin{tabular}{c|c|c|c}
\hline Ano & Domićlios na amostra & Domicílios na população & Pessoas na população (milhões) \\
\hline 2004 & 107.999 & 49.447 .777 & 173,68 \\
2009 & 115.260 & 55.674 .445 & 182,03 \\
Total & 223.259 & 105.122 .222 & 355,70 \\
\hline
\end{tabular}

Foram estimados modelos de lógite para avaliar o efeito de vários condicionantes sobre a insegurança alimentar medida pela EBIA. Sendo $P_{i}$ a probabilidade de o $i$-ésimo domicílio pertencer a certa categoria de insegurança alimentar e indicando por $x_{h i}$, com $h=1, \ldots, k$, as $k$ variáveis explanatórias consideradas, o modelo de lógite é

$$
P_{i}=\frac{1}{1+\exp \left(-y_{i}\right)}
$$

com

$$
y_{i}=\alpha+\beta_{1} x_{1 i}+\beta_{2} x_{2 i}+\ldots+\beta_{k} x_{k i}
$$

De (1) segue-se que

$$
y_{i}=\ln \frac{P_{i}}{1-P_{i}}
$$

que é o lógite do $i$-ésimo domicílio.

Quando o valor de $x_{h}$ aumenta de 1, mantidas constantes as demais variáveis explanatórias, o valor do lógite aumenta de $\beta_{h}$, o que corresponde a multiplicar a relação $P_{i} /\left(1-P_{i}\right)$ por $\exp \left(\beta_{h}\right)$, que é a odds ratio associada a $x_{h}$.

$$
\text { Se } P_{0} /\left(1-P_{0}\right) \text { é a odds inicial e } P_{1} /\left(1-P_{1}\right) \text { é }
$$

a odds após o aumento de uma unidade em $x_{h}$, a odds ratio associada a $x_{h}$ é

$$
\exp \left(\beta_{h}\right)=\frac{\frac{P_{1}}{1-P_{1}}}{\frac{P_{0}}{1-P_{0}}}
$$

A vantagem do modelo de lógite (1) é que ele permite estimar como uma determinada variável $x_{h}$ afeta a probabilidade $P$, quando as demais variáveis permanecem constantes.

As estimativas dos parâmetros foram obtidas pelo método da máxima verossimilhança, levando em consideração o fator de expansão (peso) de cada domicílio, fornecido pelo IBGE 4 .

\section{RESULTADOS BÁSICOS}

A Tabela 2 mostra as mudanças, entre 2004 e 2009, na incidência dos diferentes graus de insegurança alimentar. Resultados semelhantes são apresentados na publicação do IBGE sobre Segurança Alimentar 2004/2009 [23]. Os números são um pouco diferentes porque aqui foram utilizados os fatores de expansão atualizados e uma amostra depurada. Confirma-se que "houve crescimento do percentual de insegurança leve e redução dos percentuais de insegurança alimentar moderada e grave" (IBGE, p. 35) [23].

Apesar de o número de domicílios na população analisada ter crescido $12,6 \%$, o número daqueles com insegurança moderada se reduz em $40,2 \%$ e o número daqueles com insegurança grave diminui $12,4 \%$.

A Tabela 3 apresenta dados semelhantes considerando, agora, o número de pessoas residentes, em lugar do número de domicílios. Como o número de pessoas por domicílio é menor naqueles com segurança alimentar, a porcentagem de pessoas com segurança

\footnotetext{
4 As variáveis utilizadas são descritas no Apêndice A e no Apêndice B comenta-se como os resultados estatísticos são afetados pela estrutura complexa da amostra da PNAD.
} 
alimentar é menor do que a respectiva porcentagem de domicílios.

Tabela 2. Número e porcentagem de domicílios conforme categorias de segurança/insegurança alimentar no Brasil, em 2004 e 2009

\begin{tabular}{|c|c|c|c|c|c|c|}
\hline \multirow[b]{2}{*}{$\begin{array}{l}\text { Segurança/ } \\
\text { Insegurança } \\
\text { alimentar }\end{array}$} & \multicolumn{2}{|c|}{2004} & \multicolumn{2}{|c|}{2009} & \multicolumn{2}{|c|}{ Variação de 2004 a 2009} \\
\hline & $\begin{array}{c}\text { Domicílios } \\
\text { (milhões) }\end{array}$ & $\%$ & $\begin{array}{l}\text { Domicílios } \\
\text { (milhões) }\end{array}$ & $\%$ & $\begin{array}{c}\text { no no } \\
(\%)\end{array}$ & $\begin{array}{c}\text { na } \\
\text { porcentagem } \\
(\%)\end{array}$ \\
\hline Com segurança & 32,31 & 65,3 & 38,89 & 69,9 & 20,3 & 6,9 \\
\hline Insegurança leve & 7,94 & 16,1 & 10,43 & 18,7 & 31,3 & 16,6 \\
\hline Insegurança & 6,08 & 12,3 & 3,63 & 6,5 & $-40,2$ & $-46,9$ \\
\hline Insegurança grave & 3,11 & 6,3 & 2,73 & 4,9 & $-12,4$ & $-22,2$ \\
\hline Total & 49,45 & 100,0 & 55,67 & 100,0 & 12,6 & - \\
\hline
\end{tabular}

Tabela 3. Número e porcentagem de pessoas ${ }^{1}$ conforme categorias de segurança/insegurança alimentar do seu domicílio, no Brasil, em 2004 e 2009

\begin{tabular}{l|c|c|c|c|c|c}
\hline \multirow{2}{*}{$\begin{array}{c}\text { Segurança/ } \\
\text { Insegurança } \\
\text { alimentar }\end{array}$} & \multicolumn{2}{c|}{$\mathbf{2 0 0 4}$} & \multicolumn{2}{c|}{ 2009 } & \multicolumn{2}{c}{ Variação de 2004 a 2009 } \\
\cline { 2 - 7 } & $\begin{array}{c}\text { Pessoas } \\
\text { (milhões) }\end{array}$ & $\mathbf{\%}$ & $\begin{array}{c}\text { Pessoas } \\
\text { (milhões) }\end{array}$ & $\mathbf{\%}$ & $\begin{array}{c}\text { na no } \\
\mathbf{( \% )}\end{array}$ & $\begin{array}{c}\text { norcentagem } \\
\text { (\%) }\end{array}$ \\
\hline Com segurança & 104,65 & 60,3 & 119,64 & 65,7 & 14,3 & 9,1 \\
Insegurança leve & 31,27 & 18,0 & 38,22 & 21,0 & 22,2 & 16,6 \\
Insegurança moderada & 24,58 & 14,1 & 13,58 & 7,5 & $-44,8$ & $-47,3$ \\
Insegurança grave & 13,17 & 7,6 & 10,58 & 5,8 & $-19,6$ & $-23,4$ \\
Total & 173,68 & 100,0 & 182,03 & 100,0 & 4,8 & - \\
\hline
\end{tabular}

${ }^{1}$ Exclusive pessoas cuja condição no domicílio era pensionista, empregado doméstico ou parente do empregado doméstico.

Agregando insegurança alimentar moderada e grave, verifica-se que a proporção de domicilios nessa condição cai de 18,6\% em 2004 para 11,4\% em 2009, e a respectiva proporção de pessoas residentes cai de $21,7 \%$ em 2004 para 13,3\% em 2009.

Posteriormente será discutido o aumento da insegurança alimentar leve. A seguir analisamos as mudanças ocorridas entre 2004 e 2009 que devem ter contribuído para a redução da insegurança alimentar, tendo em vista os seus condicionantes mais importantes.

Como mostra a Tabela 4, o valor real médio da Renda Domiciliar Per Capita (RDPC) na população analisada cresceu $28,0 \%$ de 2004 a 2009. O valor da mediana da distribuição cresceu bem mais $(41,1 \%)$, pois houve redução da assimetria da distribuição, acompanhando a redução da desigualdade. Enquanto a renda média real dos $40 \%$ mais pobres aumenta $44,7 \%$, para os $10 \%$ com maiores rendas esse aumento é de
20,6\%. O índice de Gini da distribuição da RDPC cai de 0,566 em 2004 para 0,536 em 2009.

Adotando o procedimento usual dos estudos sobre distribuição da renda no Brasil, a RDPC é calculada como o cociente entre a renda domiciliar e o número de pessoas do domicílio, excluindo as pessoas cuja condição no domicílio é de pensionista, empregado doméstico (residente no domicílio do empregador) ou parente de empregado doméstico. Note-se, entretanto, que a EBIA foi aplicada pelo IBGE considerando todos os membros do domicílio. Inclusive ao distinguir domicílios com ou sem menores de 18 anos, foram consideradas pessoas daquelas 3 categorias. Essa inconsistência entre a aplicação da escala de insegurança alimentar e a definição da RDPC no que se refere ao tamanho do domicílio afeta muito pouco os resultados, pois na população analisada apenas $0,75 \%$ dos domićlios possuem alguma pessoa daquelas 3 categorias e essas pessoas representam apenas $0,28 \%$ do total. 
Tabela 4. Mudanças em condicionantes da segurança ou insegurança alimentar no Brasil ${ }^{1}$ entre 2004 e 2009

\begin{tabular}{|c|c|c|}
\hline Variável & 2004 & 2009 \\
\hline $\mathrm{RDPC}^{2}$ média & 498,7 & 638,4 \\
\hline $\mathrm{RDPC}^{2}$ mediana & 269,3 & 380,0 \\
\hline $\mathrm{RDPC}^{2}$ média dos $40 \%$ mais pobres & 117,4 & 169,9 \\
\hline RDPC $^{2}$ média dos $10 \%$ mais ricos & $2.241,6$ & $2.703,4$ \\
\hline Índice de Gini da RDPC & 0,566 & 0,536 \\
\hline$\%$ de pessoas com RDPC $\leq 250$ & 46,7 & 33,4 \\
\hline$\%$ de domicílios rurais & 15,8 & 15,4 \\
\hline \% de domicílios com luz elétrica & 96,8 & 98,8 \\
\hline$\%$ de domicílios com água canalizada ${ }^{3}$ & 89,3 & 93,0 \\
\hline$\%$ de domić́lios com esgoto 4 & 68,4 & 71,8 \\
\hline média de pessoas por domicílio & 3,5 & 3,3 \\
\hline média de pessoas por cômodo & 0,69 & 0,62 \\
\hline PR5: Escolaridade média & 6,2 & 7,0 \\
\hline Escolaridade mediana & 5,0 & 7,0 \\
\hline$\%$ atividade agrícola & 16,0 & 13,6 \\
\hline
\end{tabular}

1 Utilizando uma amostra depurada das PNADs de 2004 e 2009, considerando apenas domicílios com renda positiva.

2 Renda Domiciliar Per Capita (RDPC), em reais de set.-out. de 2009, atualizando os valores correntes de 2004 com base na média geométrica dos valores do Índice Nacional de Preços ao Consumidor (INPC) de setembro e outubro.

3 Tem água canalizada em pelo menos um cômodo.

${ }^{4}$ Escoadouro do banheiro ou sanitário ligado a rede coletora e/ou fossa séptica.

5 Pessoa de Referência do domicílio.

Uma vez que a renda é o principal condicionante da insegurança alimentar medida pela EBIA [2], o substancial aumento da RDPC média deve ter contribuído para reduzir a insegurança alimentar. Além disso, o crescimento mais intenso da renda dos mais pobres, simultaneamente à redução da desigualdade da distribuição da renda, deve ter contribuído ainda mais para a redução da insegurança alimentar.

O aumento da proporção de domicílios com luz elétrica, com água canalizada e com esgoto, além da redução do número médio de pessoas por cômodo, também indicam melhoras nas condições de vida, que estão associadas com a redução da insegurança alimentar.

Verifica-se, na Tabela 4, que houve aumento nas escolaridades média e mediana das pessoas de referência dos domicilios, o que também deve ter contribuído para a redução da insegurança alimentar.

\section{Modelos de lógite para insegurança alimentar em 2004 e 2009}

Tendo em vista deixar clara a limitação de uma análise estatística sem controles, vamos considerar, inicialmente, apenas a localização rural ou urbana do domicílio como variável explanatória, utilizando a amostra agregada das PNADs de 2004 e 2009.

A Tabela 5 mostra os dados básicos referentes à incidência de Insegurança Alimentar Moderada ou Grave (IAMG) nessa amostra e na população correspondente. Observa-se que ela é maior na área rural 5 .

Analisando os dados da amostra como uma tabela de contingência $2 \times 2$, verifica-se que a incidência de IAMG é significativamente maior na área rural. $\mathrm{O}$ teste usual de qui-quadrado é igual a 618,5, com probabilidade caudal inferior a $0,01 \% 0^{6}$.

Uma análise essencialmente equivalente pode ser feita estimando um modelo de lógite em que a variável dependente é a probabilidade de o domicilio apresentar IAMG e a única variável explanatória é uma binária que assume valor zero para domicílios urbanos e valor 1 para domicílios rurais.

${ }^{5}$ O próprio IBGE ${ }^{[23]}$ (p. 35), ao fazer uma análise descritiva dos resultados da pesquisa, ressalta que a área rural apresentou prevalências domiciliares de insegurança alimentar superiores às verificadas na área urbana.

${ }^{6}$ Levando em consideração o fator de expansão de cada domicílio, tomando o cuidado para que isso não afete a frequência total, o valor do qui-quadrado se torna igual a 1.056,2. 
Como a proporção de IAMG na área urbana é 0,137 e na área rural é 0,204 (ver última coluna da tabela 5), a odds ratio, de acordo com a expressão (4), é

$$
\frac{\frac{0,204}{1-0,204}}{\frac{0,137}{1-0,137}}=1,614
$$

e a estimativa do único parâmetro $\beta$ do modelo é $\ln (1,614)=0,4787$. Fazendo cálculos com maior número de algarismos, no computador obtivemos uma estimativa igual a 0,4786 , como consta na Tabela 6 . Novamente, o teste da hipótese de nulidade do parâmetro tem probabilidade caudal inferior a $0,01 \%$.

Há uma relação estatisticamente muito clara entre residência rural e maior incidência de IAMG. Podemos concluir que a residência rural aumenta a insegurança alimentar? Não, pois se trata de um caso de relação espúria, associada com uma terceira variável. A insegurança alimentar é maior em domicílios rurais porque eles são, em média, mais pobres. Basta controlar o efeito da RDPC para que a estimativa do coeficiente da binária para "rural" se torne negativa, como mostra a Tabela 7.

Tabela 5. Incidências de Insegurança Alimentar Moderada ou Grave (IAMG) na amostra depurada e na população, agregando os dados das PNADs de 2004 e 2009, conforme situação do domicílio

\begin{tabular}{l|c|c|c|c|c}
\hline \multirow{2}{*}{$\begin{array}{c}\text { Situação do } \\
\text { domicílio }\end{array}$} & \multicolumn{3}{|c|}{ Domicílios na amostra } & \multicolumn{2}{c}{ Domicílios na população } \\
\cline { 2 - 6 } & Total & Com IAMG & \% & Total (milhões) & \% com IAMG \\
\hline Urbana & 190.137 & 28.927 & 15,2 & 88,7 & 13,7 \\
Rural & 33.122 & 6.838 & 20,6 & 16,4 & 20,4 \\
Total & 223.259 & 35.765 & 16,0 & 105,1 & 14,8 \\
\hline
\end{tabular}

Tabela 6. Modelo de lógite para a probabilidade de Insegurança Alimentar Moderada ou Grave (IAMG) estimado com a amostra agregada de 2004 e 2009, considerando apenas a situação urbana ou rural do domicílio

\begin{tabular}{l|c|c|c|c}
\hline \multicolumn{1}{c}{ Variável } & $\begin{array}{c}\text { Estimativa do } \\
\text { parâmetro }\end{array}$ & $\begin{array}{c}\text { Estimativa do } \\
\text { desvio padrão }\end{array}$ & $\begin{array}{c}\text { Probabilidade } \\
\text { Caudal }^{1}\end{array}$ & Odds ratio \\
\hline Constante & $-1,8288$ & 0,0067 & $*$ & - \\
Área rural & 0,4786 & 0,0148 & $*$ & 1,614 \\
\hline
\end{tabular}

$1 \mathrm{O}$ asterisco indica que a probabilidade caudal do teste da hipótese de nulidade do parâmetro é inferior a $0,01 \%$.

Tabela 7. Modelo de lógite para a probabilidade de Insegurança Alimentar Moderada ou Grave (IAMG) estimado com a amostra agregada de 2004 e 2009, considerando a situação urbana ou rural do domićlio e a Renda Domiciliar Per Capita (RDPC)

\begin{tabular}{|c|c|c|c|c|}
\hline Variável & $\begin{array}{c}\text { Estimativa do } \\
\text { parâmetro }\end{array}$ & $\begin{array}{l}\text { Estimativa do } \\
\text { desvio padrão }\end{array}$ & $\begin{array}{c}\text { Probabilidade } \\
\text { caudal }^{1}\end{array}$ & Odds ratio \\
\hline Constante & $-0,8702$ & 0,3253 & $0,8 \%$ & - \\
\hline Área rural & $-0,3048$ & 0,0168 & $*$ & 0,737 \\
\hline $2 \ln (\mathrm{RDPC})$ & 1,7520 & 0,2032 & $*$ & - \\
\hline Quadrado de $\ln (\mathrm{RDPC})$ & $-0,4378$ & 0,0420 & $*$ & - \\
\hline Cubo de $\ln (\mathrm{RDPC})$ & 0,0180 & 0,0028 & $*$ & - \\
\hline
\end{tabular}

${ }^{1}$ Probabilidade caudal do teste da hipótese de nulidade do parâmetro. $\mathrm{O}$ asterisco indica que ela é inferior a $0,01 \%$.

2 Logaritmo neperiano da Renda Domiciliar Per Capita. 
Note-se que foi necessário incluir, como variáveis explanatórias, não apenas o logaritmo da RDPC, mas também o seu quadrado e o seu cubo. O fato de os parâmetros do quadrado e do cubo serem estatisticamente diferentes de zero mostra que seria inapropriado considerar apenas o efeito linear do logaritmo da RDPC.

A função cúbica estimada tem ponto de máximo com RDPC próxima de $\mathrm{R} \$ 11$ e ponto de mínimo para RDPC de quase 1 milhão de reais. Tratase, portanto, de uma função continuamente decrescente no intervalo relevante, mostrando que a IAMG tende a diminuir com o aumento da renda?

Contrariando o que sugeriam os resultados apresentados nas Tabelas 5 e 6 , a Tabela 7 mostra que, dado o nível de renda, a situação rural do domicílio contribui para reduzir a probabilidade de IAMG.

A Tabela 8 mostra os resultados obtidos com um modelo de lógite para a probabilidade de um domicílio brasileiro ter IAMG, utilizando a amostra agregada dos dados de 2004 e 2009. O Apêndice A apresenta a descrição das variáveis utilizadas. Além de quase todas as variáveis explanatórias já consideradas no trabalho anterior [2], incluímos uma variável binária para captar o efeito do $a^{8} \mathrm{~s}^{8}$. Essa variável assume valor 0 para 2004 e valor 1 para 2009. Na Tabela 8 o respectivo coeficiente é negativo e estatisticamente significativo, indicando que a redução da IAMG de 2004 a 2009 foi maior do que aquela que seria considerada "explicável", por meio do modelo, em função das mudanças em todas as demais variáveis incluídas. Voltaremos a esse tema adiante.

$\mathrm{O}$ fato de a grande maioria dos coeficientes estimados ser estatisticamente diferente de zero mostra a relevância da equação estimada. A qualidade do ajustamento pode ser avaliada pelo coeficiente $c$, baseado na proporção de pares concordantes, isto é, pares de observações para os quais a ordenação conforme as probabilidades estimadas é igual à ordenação conforme valores observados da variável dependente. Para a equação da Tabela 8 o valor de $c$ é 0,835 .

${ }^{7}$ Nos dados da PNAD analisados, o maior valor registrado para a RDPC é $\mathrm{R} \$ 94.670$.

${ }^{8}$ No artigo anterior foi usada uma variável binária que era igual a 1 quando mais de $25 \%$ da renda domiciliar provinha de aposentadorias e pensões. Neste artigo essa variável foi substituída pela fração da renda domiciliar proveniente de aposentadorias e pensões pagas pelo poder público.
Pode-se verificar que a função cúbica no logaritmo da RDPC na Tabela 8 tem ponto de máximo quando a RDPC é R \$11,2 e ponto de mínimo quando a RDPC é 3,6 milhões. Trata-se, portanto, de uma função continuamente decrescente no intervalo relevante.

Para avaliar o efeito do crescimento da renda na redução da IAMG, vamos comparar duas situações hipotéticas. Para isso definimos como base um domicílio, em 2004, com 4 moradores em área urbana do Nordeste, com luz elétrica, água encanada, esgoto, 0,7 pessoas por cômodo, com pelo menos uma pessoa com menos de 18 anos, sem renda proveniente de aposentadorias e pensões pagas pelo poder público e cuja pessoa de referência tem 55 anos ou menos de idade, é preta ou parda, tem 5 anos de escolaridade e trabalha por conta própria em atividade não agrícola. Se nesse domicílio-base a RDPC for R $\$ 250$, utilizando os coeficientes estimados da Tabela 8 verifica-se que sua probabilidade de IAMG é 19,1\%. Essa probabilidade cai para 3,1\% se a RDPC for $\mathrm{R} \$ 1.000$, para apenas $1,0 \%$ se a $\mathrm{RDPC}$ for $\mathrm{R} \$ 2.000$ e se torna praticamente desprezível (menos de $0,3 \%$ ) se a RDPC for R $\$ 5.000$.

É importante ter em mente que em levantamentos domiciliares, como a PNAD, ocorrem erros substanciais na declaração da renda domiciliar, com tendência de subestimação do seu valor. É provável que parte do efeito da renda seja captada por outras variáveis incluídas no modelo, como a escolaridade e o número de pessoas por cômodo (com sinal oposto). O coeficiente negativo de "empregador" provavelmente está associado à maior subdeclaração da renda das pessoas com essa posição na ocupação.

Consideremos, novamente, nosso domicíliobase com RDPC igual a $R \$ 250$, com probabilidade estimada de IAMG igual a 19,1\%. Se ele estivesse no Sudeste ou no Sul, e não no Nordeste, essa probabilidade seria $12,8 \%$. No Centro-Oeste e no Norte a probabilidade de IAMG seria 14,1\% e 17,6\%, respectivamente. Se a pessoa de referência fosse branca, em lugar de preta ou parda, a probabilidade estimada de IAMG cairia de $19,1 \%$ para $15,1 \%$. Se a pessoa de referência do domicílio fosse um empregado com carteira, e não um trabalhador por conta própria, a probabilidade estimada de IAMG cairia de 19,1\% para $16,1 \%$. Mas se ele fosse um empregado sem carteira de trabalho assinada a probabilidade subiria para $21,7 \%$. 
Tabela 8. Modelo de lógite para a probabilidade de um domicílio ter Insegurança Alimentar Moderada ou Grave (IAMG). Brasil, 2004 e 2009

\begin{tabular}{|c|c|c|c|c|c|}
\hline \multicolumn{2}{|c|}{ Variável } & $\begin{array}{c}\text { Estimativa do } \\
\text { parâmetro }\end{array}$ & $\begin{array}{l}\text { Estimativa do } \\
\text { desvio padrão }\end{array}$ & $\begin{array}{c}\text { Probabilidade } \\
\text { caudal }^{1}\end{array}$ & Odds ratio \\
\hline \multicolumn{2}{|c|}{ Constante } & $-0,5430$ & 0,3489 & $12,0 \%$ & - \\
\hline \multicolumn{2}{|c|}{ Ano de 2009} & $-0,2839$ & 0,0141 & $*$ & 0,753 \\
\hline \multicolumn{2}{|c|}{${ }^{2} \ln (\mathrm{RDPC})$} & 1,5652 & 0,2175 & $*$ & - \\
\hline \multicolumn{2}{|c|}{ Quadrado de $\ln ($ RDPC) } & $-0,3758$ & 0,0453 & $*$ & - \\
\hline \multicolumn{2}{|c|}{ Cubo de $\ln (\mathrm{RDPC})$} & 0,0143 & 0,0031 & $*$ & - \\
\hline \multicolumn{2}{|c|}{$\mathrm{PR}^{3}$ Escolaridade } & $-0,0616$ & 0,0021 & $*$ & 0,940 \\
\hline \multicolumn{2}{|c|}{ PR 3 Mulher } & 0,3951 & 0,0169 & $*$ & 1,485 \\
\hline \multicolumn{2}{|c|}{$\mathrm{PR}^{3}>55$ anos } & $-0,0274$ & 0,0211 & $19,6 \%$ & 0,973 \\
\hline \multicolumn{2}{|c|}{$\mathrm{PR}^{3}$ preto ou pardo } & 0,2836 & 0,0153 & $*$ & 1,328 \\
\hline \multicolumn{2}{|c|}{$\mathrm{PR}^{3}$ amarelo } & $-0,0759$ & 0,1505 & $61,4 \%$ & 0,927 \\
\hline \multicolumn{2}{|c|}{ Pessoas/cômodo } & 0,5790 & 0,0198 & $*$ & 1,784 \\
\hline & 0,5855 & 0,0332 & $*$ & 1,796 \\
\hline \multicolumn{2}{|c|}{ Pessoas/domicílio 1} & 0,2761 & 0,0265 & $*$ & 1,318 \\
\hline & 0,0831 & 0,0214 & * & 1,087 \\
\hline \multicolumn{2}{|r|}{5 ou 6} & 0,0258 & 0,0213 & $22,7 \%$ & 1,026 \\
\hline \multicolumn{2}{|r|}{$\geq 7$} & 0,0829 & 0,0316 & $0,9 \%$ & 1,086 \\
\hline \multicolumn{2}{|c|}{ Luz elétrica } & $-0,0658$ & 0,0381 & $8,4 \%$ & 0,936 \\
\hline \multicolumn{2}{|c|}{ Água canalizada 4} & $-0,3330$ & 0,0231 & $*$ & 0,717 \\
\hline \multicolumn{2}{|c|}{ Esgoto $^{5}$} & $-0,0442$ & 0,0168 & $0,9 \%$ & 0,957 \\
\hline \multirow[t]{5}{*}{ Região $^{6}$} & Norte & $-0,0998$ & 0,0248 & $*$ & 0,905 \\
\hline & $\mathrm{MG}+\mathrm{ES}+\mathrm{RJ}$ & $-0,4696$ & 0,0205 & * & 0,625 \\
\hline & SP & $-0,4740$ & 0,0231 & $*$ & 0,623 \\
\hline & Sul & $-0,4760$ & 0,0258 & * & 0,621 \\
\hline & Centro-Oeste & $-0,3608$ & 0,0290 & * & 0,697 \\
\hline \multicolumn{2}{|c|}{ Área rural } & $-0,5889$ & 0,0231 & $*$ & 0,555 \\
\hline \multicolumn{2}{|c|}{ Setor agrícola } & $-0,1633$ & 0,0225 & $*$ & 0,849 \\
\hline \multicolumn{2}{|c|}{ PO(7) Mil. ou func.públ. } & 0,1097 & 0,0454 & $1,6 \%$ & 1,116 \\
\hline \multicolumn{2}{|c|}{ Sem carteira ${ }^{8}$} & 0,3704 & 0,0246 & $*$ & 1,448 \\
\hline \multicolumn{2}{|c|}{ Doméstico } & 0,1841 & 0,0350 & * & 1,202 \\
\hline \multicolumn{2}{|c|}{ Conta própria } & 0,2070 & 0,0227 & $*$ & 1,230 \\
\hline \multicolumn{2}{|c|}{ Empregador } & $-0,4007$ & 0,0672 & * & 0,670 \\
\hline \multicolumn{2}{|c|}{ Inativos e outros } & 0,1180 & 0,0260 & $*$ & 1,125 \\
\hline Fração d & apos. e pensões 9 & $-0,0286$ & 0,0287 & $31,8 \%$ & 0,972 \\
\hline Tem pes & oa $<18$ anos & $-0,6436$ & 0,0222 & $*$ & 0,525 \\
\hline
\end{tabular}

${ }^{1}$ Probabilidade caudal do teste da hipótese de nulidade do parâmetro. O asterisco assinala os casos em que essa probabilidade é inferior a $0,1 \%$.

${ }^{2}$ Logaritmo neperiano da Renda Domiciliar Per Capita (RDPC).

${ }^{3}$ Pessoa de referência do domicílio, adotando como base o homem branco com até 55 anos de idade.

4 Tem água canalizada em pelo menos um cômodo.

${ }^{5}$ Escoadouro do banheiro ou sanitário ligado a rede coletora e/ou fossa séptica.

${ }^{6}$ A Região Nordeste é adotada como base.

7 Posição na ocupação da pessoa de referência do domicílio, adotando como base os empregados com carteira.

${ }^{8}$ Empregados sem carteira ou sem declaração de carteira.

${ }^{9}$ Fração da renda domiciliar constituída por aposentadorias e pensões pagas pelo poder público.

Note-se que os resultados são coerentes com a ideia de a segurança alimentar ser favorecida tanto pela renda maior como pela estabilidade ou regularidade dessa renda, fazendo com que a probabilidade de insegurança alimentar seja maior quando a pessoa de referência do domicílio é um empregado sem carteira de trabalho assinada ou um trabalhador por conta própria, do que quando ele é um empregado com carteira.

Há, no modelo, variáveis explanatórias cujo coeficiente não pode ser analisado separadamente, pressupondo que todas as demais variáveis 
explanatórias sejam mantidas constantes. Isso é óbvio no caso do logaritmo da RDPC, cuja variação implica a variação simultânea do seu quadrado e do seu cubo. Mas deve-se notar, também, que a presença de crianças e adolescentes no domicílio (a última variável da Tabela 8) está associada a maior número de pessoas no domicílio, maior número de pessoas por cômodo e menor RDPC. Em um modelo de lógite para IAMG incluindo como variável explanatória apenas a presença, no domicílio, de pessoa com menos de 18 anos, a estimativa do coeficiente é 0,4947 , positiva e estatisticamente significativa, embora na Tabela 8 o coeficiente da mesma variável seja negativo e estatisticamente significativo. Utilizando um modelo sem as variáveis referentes ao número de pessoas por domicílio e número de pessoas por cômodo, o coeficiente de "presença de pessoa com menos de 18 anos" é -0,6489, negativo e significativo, se for usado o logaritmo da RDPC, como na Tabela 8, mas se torna igual a 0,2884 , positivo e significativo, se for usado o logaritmo da renda domiciliar (em lugar da RDPC). Isso mostra que a estimação de um efeito positivo da presença de pessoa com menos de 18 anos sobre a probabilidade de IAMG se deve, essencialmente, a não levar em consideração a correlação negativa entre essa presença e a RDPC.

A Tabela 9 mostra os resultados obtidos com um modelo de lógite para Insegurança Alimentar Grave. As variáveis explanatórias são as mesmas consideradas na Tabela 8, para IAMG. O coeficiente $c$ para essa equação é 0,843 .

Embora o coeficiente do cubo do logaritmo da RDPC não seja estatisticamente diferente de zero, considerou-se preferível manter o mesmo modelo usado para IAMG. Verifica-se que a função cúbica é decrescente no intervalo relevante, a partir de uma $\mathrm{RDPC}$ de $\mathrm{R} \$ 7,5$.

Comparando as Tabelas 8 e 9, observa-se que, além do coeficiente para "Ano de 2009", apenas dois outros mudam de sinal: o coeficiente para pessoas de referência amarela, que não é estatisticamente significativo, e o coeficiente que capta o efeito de o domicílio estar na Região Norte, em comparação com o Nordeste.
Consideremos, novamente o nosso domiciliobase. Se sua RDPC for R $\$ 250$, a probabilidade de que sofra de insegurança alimentar grave, de acordo com os resultados apresentados na Tabela 9, é 4,4\%. Essa probabilidade se torna desprezível (menos de $0,03 \%$ ) se a RDPC for igual a $\mathrm{R} \$ 5.000$.

Se o domicílio estiver nas Regiões Sul, Sudeste ou Centro-Oeste, e não no Nordeste, a estimativa da probabilidade de insegurança alimentar grave cai de 4,4\% para cerca de 3,4\%. Se a pessoa de referência for um empregado com carteira de trabalho assinada, e não um trabalhador por conta própria, a probabilidade cai de $4,4 \%$ para 3,3\%; mas ela sobe para $5,3 \%$ no caso de um empregado sem carteira. E se a pessoa de referência for branco, essa probabilidade cai de $4,4 \%$ para $3,2 \%$.

É intrigante constatar que o coeficiente de "Ano de 2009" é negativo no modelo para IAMG (Tabela 8) e positivo no modelo para insegurança alimentar grave (Tabela 9), sendo estatisticamente significativo nos dois casos. Esse coeficiente representa a mudança ocorrida na insegurança alimentar entre os dois anos que não é "explicada" por todas as demais variáveis explanatórias incluídas no modelo.

a) Um coeficiente negativo para "Ano de 2009" significa que a redução na insegurança alimentar foi maior do que o "explicado" pela variação das demais variáveis. Isso pode ocorrer devido à redução da desigualdade. A renda aumentou mais para os pobres, isto é, para os domicílios mais vulneráveis à insegurança alimentar.

b) Um coeficiente positivo para "Ano de 2009" indica que a redução da insegurança alimentar foi menor do que o "explicado" pela variação das demais variáveis. Isso pode ocorrer devido ao fato de as pessoas estarem mais conscientes do seu "direito" à segurança alimentar, e com maior tendência de se manifestar contra situações adversas, aumentando a probabilidade de denunciarem eventuais situações de insegurança alimentar.

No caso da IAMG, deve ter predominado o tipo de efeito descrito em (a) e no caso da insegurança grave aparentemente foi predominante o tipo de efeito descrito em (b). 
Tabela 9. Modelo de lógite para a probabilidade de um domicílio ter insegurança alimentar grave. Brasil, 2004 e 2009

\begin{tabular}{|c|c|c|c|c|c|}
\hline & Variável & $\begin{array}{c}\text { Estimativa do } \\
\text { parâmetro }\end{array}$ & $\begin{array}{l}\text { Estimativa do } \\
\text { desvio padrão }\end{array}$ & $\begin{array}{c}\text { Probabilidade } \\
\text { caudal }^{1}\end{array}$ & Odds ratio \\
\hline \multicolumn{2}{|c|}{ Constante } & $-1,0314$ & 0,3966 & $0,9 \%$ & - \\
\hline \multicolumn{2}{|c|}{ Ano de 2009} & 0,1488 & 0,0208 & $*$ & 1,160 \\
\hline \multicolumn{2}{|c|}{${ }^{2} \ln (\mathrm{RDPC})$} & 0,7716 & 0,2623 & $0,3 \%$ & - \\
\hline \multicolumn{2}{|c|}{ Quadrado de $\ln (\mathrm{RDPC})$} & $-0,1951$ & 0,0579 & $*$ & - \\
\hline \multicolumn{2}{|c|}{ Cubo de $\ln (\mathrm{RDPC})$} & 0,0014 & 0,0042 & $74,2 \%$ & - \\
\hline \multicolumn{2}{|c|}{$\mathrm{PR}^{3}$ Escolaridade } & $-0,0769$ & 0,0032 & $*$ & 0,926 \\
\hline \multicolumn{2}{|c|}{$\mathrm{PR}^{3}$ Mulher } & 0,3566 & 0,0246 & $*$ & 1,428 \\
\hline \multicolumn{2}{|c|}{$\mathrm{PR}^{3}>55$ anos } & $-0,0659$ & 0,0306 & $3,1 \%$ & 0,936 \\
\hline \multicolumn{2}{|c|}{$\mathrm{PR}^{3}$ preto ou pardo } & 0,3253 & 0,0233 & $*$ & 1,384 \\
\hline \multicolumn{2}{|c|}{$\mathrm{PR}^{3}$ amarelo } & 0,2250 & 0,2138 & $29,3 \%$ & 1,252 \\
\hline \multicolumn{2}{|c|}{ Pessoas/cômodo } & 0,4961 & 0,0212 & $*$ & 1,642 \\
\hline \multicolumn{2}{|c|}{ Pessoas/domicílio 1} & 0,9627 & 0,0475 & $*$ & 2,619 \\
\hline \multicolumn{2}{|c|}{2} & 0,3486 & 0,0400 & $*$ & 1,417 \\
\hline \multicolumn{2}{|c|}{3} & 0,0348 & 0,0336 & $30,1 \%$ & 1,035 \\
\hline \multicolumn{2}{|c|}{5 ou 6} & 0,0400 & 0,0318 & $20,7 \%$ & 1,041 \\
\hline \multicolumn{2}{|c|}{$\geq 7$} & 0,2718 & 0,0410 & $*$ & 1,312 \\
\hline \multicolumn{2}{|c|}{ Luz elétrica } & $-0,0331$ & 0,0487 & $49,7 \%$ & 0,967 \\
\hline \multicolumn{2}{|c|}{ Água canalizada ${ }^{4}$} & $-0,3753$ & 0,0304 & $*$ & 0,687 \\
\hline \multicolumn{2}{|c|}{ Esgoto $^{5}$} & $-0,0623$ & 0,0244 & $1,1 \%$ & 0,940 \\
\hline \multirow[t]{5}{*}{ Região ${ }^{6}$} & Norte & 0,0779 & 0,0336 & $2,1 \%$ & 1,081 \\
\hline & $\mathrm{MG}+\mathrm{ES}+\mathrm{RJ}$ & $-0,3774$ & 0,0314 & $*$ & 0,686 \\
\hline & $\mathrm{SP}$ & $-0,2817$ & 0,0353 & $*$ & 0,754 \\
\hline & Sul & $-0,3093$ & 0,0402 & $*$ & 0,734 \\
\hline & Centro-Oeste & $-0,2682$ & 0,0445 & $*$ & 0,765 \\
\hline \multicolumn{2}{|c|}{ Área rural } & $-0,6212$ & 0,0327 & $*$ & 0,537 \\
\hline \multicolumn{2}{|c|}{ Setor agrícola } & $-0,1796$ & 0,0317 & $*$ & 0,836 \\
\hline \multicolumn{2}{|c|}{ PO(7) Mil. ou func. públ. } & 0,1002 & 0,0797 & $20,9 \%$ & 1,105 \\
\hline \multicolumn{2}{|c|}{$\begin{array}{l}\text { PO(7) Mil. ou func. públ. } \\
\text { Sem carteira }\end{array}$} & 0,5042 & 0,0385 & $*$ & 1,656 \\
\hline \multicolumn{2}{|c|}{ Doméstico } & 0,3129 & 0,0516 & $*$ & 1,367 \\
\hline \multicolumn{2}{|c|}{ Conta própria } & 0,3046 & 0,0367 & $*$ & 1,356 \\
\hline \multicolumn{2}{|c|}{ Empregador } & $-0,3709$ & 0,1227 & $0,2 \%$ & 0,690 \\
\hline \multicolumn{2}{|c|}{ Inativos e outros } & 0,2389 & 0,0406 & $*$ & 1,270 \\
\hline \multicolumn{2}{|c|}{ Fração de apos. e pensões 9} & $-0,1079$ & 0,0417 & $1,0 \%$ & 0,898 \\
\hline Tem pes & soa $<18$ anos & $-0,7089$ & 0,0340 & $*$ & 0,492 \\
\hline
\end{tabular}

${ }_{1}^{1}$ Probabilidade caudal do teste da hipótese de nulidade do parâmetro. $\mathrm{O}$ asterisco assinala os casos em que essa probabilidade é inferior a $0,1 \%$.

2 Logaritmo neperiano da Renda Domiciliar Per Capita (RDPC).

3 Pessoa de referência do domićlio, adotando como base o homem branco com até 55 anos de idade.

4 Tem água canalizada em pelo menos um cômodo.

${ }^{5}$ Escoadouro do banheiro ou sanitário ligado a rede coletora e/ou fossa séptica.

${ }^{6}$ A Região Nordeste é adotada como base.

7 Posição na ocupação da pessoa de referência do domicílio, adotando como base os empregados com carteira.

${ }^{8}$ Empregados sem carteira ou sem declaração de carteira.

${ }^{9}$ Fração da renda domiciliar constituída por aposentadorias e pensões pagas pelo poder público.

Parece que o tipo de efeito descrito em (b) foi ainda mais intenso no caso da insegurança alimentar leve, cuja incidência aumentou de 2004 a 2009 (ver Tabela 2), apesar do crescimento da RDPC e da escolaridade. Tendo em vista o caráter subjetivo das perguntas destinadas a captar a insegurança alimentar leve, a difusão da ideia de que o governo deve auxiliar as famílias com insegurança alimentar pode ter contribuído para aumentar o número de domicílios classificados nessa categoria na PNAD, dada a 
perspectiva de obter algum benefício, como uma transferência de renda do Programa Bolsa Família.

\section{PRINCIPAIS CONCLUSÕES}

A análise conjunta dos dados sobre segurança alimentar coletados nas PNADs de 2004 e 2009 confirma resultados já obtidos da análise dos dados de 2004:

a) A renda domiciliar per capita pode ser considerada o principal determinante da insegurança alimentar. Os modelos de lógite estimados mostram que uma renda elevada torna a insegurança alimentar desprezível.

b) A estabilidade da fonte de renda contribui para a segurança alimentar. Fixados o nível de renda e outros fatores, o fato de a pessoa de referência ser um empregado com carteira de trabalho assinada faz com que a probabilidade de insegurança alimentar do domicílio seja substancialmente menor do que se ela for um empregado sem carteira de trabalho assinada.

c) A maior escolaridade também contribui para reduzir a probabilidade de insegurança alimentar.

d) Contrariamente ao sugerido por uma análise baseada em uma simples tabela de contingência entre nível de insegurança alimentar e a situação urbana ou rural do domićlio, os modelos de lógite mostram que, fixados os demais fatores, a situação rural contribui para reduzir a probabilidade de insegurança alimentar.

e) Há efeitos regionais importantes, além dos explicáveis pelas diferenças de renda e escolaridade.

De 2004 a 2009 a renda real per capita mediana aumentou mais de $40 \%$ e a escolaridade mediana das pessoas de referência dos domicílios aumentou de 5 para 7. Em função disso, seria esperada uma redução da insegurança alimentar, como efetivamente ocorreu com a insegurança alimentar moderada e com a insegurança alimentar grave. Estranhamente, a insegurança alimentar leve, como medida pela EBIA, aumentou. A provável explicação para isso é a natureza bastante subjetiva da escala, combinada com mudanças na opinião pública sobre o papel do estado na superação das eventuais dificuldades para adquirir alimentos.

\section{REFERÊNCIAS}

[1] Segall Corrêa AM, Escamilla RP, Maranha LK, Sampaio MFA (Org.). (In)Segurança alimentar no Brasil: validação de metodologia para acompanhamento e avaliação. Relatório Técnico. UNICAMP, Faculdade de Ciências Médicas; 2003.

[2] Hoffmann R. Determinantes da insegurança alimentar no Brasil: análise dos dados da PNAD de 2004. Seg Alim Nutr. 2008;15(1):49-61.

[3] Rocha C, Burlandy L, Magalhães R. (Org.). Segurança Alimentar e Nutricional: perspectivas, aprendizados e desafios para as políticas públicas. Rio de Janeiro: Editora FIOCRUZ; 2013.

[4] Maluf RS, Menezes F, Valente FL. Contribuição ao tema da segurança alimentar no Brasil. Revista Cadernos de Debate, NEPA-UNICAMP. 1996;6:66-88.

[5] Instituto de Pesquisa Econômica Aplicada (IPEA). A segurança alimentar e nutricional e o direito humano à alimentação no Brasil. Brasília: IPEA; 2002.

[6] Belik W. Perspectivas para segurança alimentar e nutricional no Brasil. Saúde Soc. 2003;12(1):12-20.

[7] Takagi MA. Implantação da política de segurança alimentar e nutricional no Brasil: seus limites e desafios [tese de doutorado]. Campinas: Instituto de EconomiaUnicamp; 2006.

[8] Conselho Nacional de Segurança Alimentar e Nutricional (CONSEA). A segurança alimentar e nutricional e o direito humano à alimentação adequada no Brasil. Brasília: CONSEA; 2010.

[9] Burity V, Franceschini T, Valente F, Recine E, Leão M, Carvalho MF. Direito, humano à alimentação adequada no contexto da segurança alimentar e nutricional. Brasília: ABRANDH; 2010.

[10] Almeida Filho N, Ramos P. (org.) Segurança alimentar: produção agrícola e desenvolvimento territorial. Campinas:Editora Alínea; 2010.

[11] Burlandy L, Magalhães R, Frozi DS. Políticas públicas de segurança alimentar e nutricional. In: Rocha C, Burlandy L, Magalhães R. (org.). Segurança Alimentar e Nutricional: perspectivas, aprendizados e desafios para as políticas públicas. Rio de Janeiro: Editora FIOCRUZ; 2013. p. 89-107. 
[12] Sen A. Poverty and famines: an essay on entitlement and deprivation. Oxford University Press; 1981.

[13] Gubert MB. Modelo preditivo de insegurança alimentar grave para os municípios brasileiros [tese de doutorado]. Brasília: Universidade de Brasília; 2009.

[14] Santos JV, Gigante DP, Domingues MR. Prevalências de insegurança alimentar em Pelotas, Rio Grande do Sul, Brasil, e estado nutricional de indivíduos que vivem nessa condição. Cad Saúde Pública. 2010;26(1):41-49.

[15] Panigassi G, Segall-Corrêa AM, Marin-León L, PérezEscamilla R, Sampaio MFA, Maranha LK. Insegurança alimentar como indicador de iniquidade: análise de inquérito populacional. Cad Saúde Pública. 2008;24(10):2376-2384.

[16] Monteiro CA. O mapa da pobreza no Brasil. Cadernos de Nutrição. 1992;4:1-6.

[17] Monteiro CA. Fome, desnutrição e pobreza: além da semântica. Saúde Soc. 2003;12(1):7-11.
[18] Monteiro CA. A dimensão da pobreza, da desnutrição e da fome no Brasil. Estud Av. 2003;17(48):7-20.

[19] Gans O. Cost effectiveness of food security systems: na assessment based on an econometric model of consumer demand. The case of Indonesia. Economics. 1999;59:96-116.

[20] Sampaio MFA, Kepple AW, Segall-Corrêa AM, Oliveira JTA, Maranha LK, Marin-Leon L, et al. (In)Segurança alimentar: experiência de grupos focais com populações rurais do Estado de São Paulo. Seg Alim Nutr. 2006;13(1):64-77.

[21] Bickel G, Nord M, Price C, Hamilton W, Cook J. Guide to measuring household food security. Revised 2000. USDA; 2000.

[22] Instituto Brasileiro de Geografia e Estatística (IBGE). Segurança alimentar: 2004. Rio de Janeiro: IBGE; 2006.

[23] Instituto Brasileiro de Geografia e Estatística (IBGE). Segurança alimentar 2004/2009. Rio de Janeiro: IBGE; 2010 . 


\section{APÊNDICE A. Descrição das variáveis utilizadas nos modelos de lógite}

A variável dependente observada é binária, assumindo valor 1 se o domicílio apresenta determinado tipo de insegurança alimentar (moderada ou grave ou apenas a grave) e valor zero em caso contrário. A variável dependente estimada é a probabilidade de o domicílio pertencer àquela(s) categoria(s) de insegurança alimentar.

Variáveis explanatórias:

1) Variável binária igual a 1 para dados de 2009 e igual a zero para dados de 2004.

2) Logaritmo neperiano da RDPC, calculada para cada domicílio dividindo a renda domiciliar pelo número de componentes, exclusive as pessoas cuja condição no domicílio era pensionista, empregado doméstico (residente no domićlio do empregador) ou parente de empregado doméstico.

3) Quadrado do logaritmo da RDPC e eventual inclusão do cubo da mesma variável.

4) Escolaridade da pessoa de referência do domićlio, variando de zero (sem instrução ou menos de 1 ano de estudo) até 14 e com valor 17 atribuído aos com 15 anos ou mais de estudo.

5) Uma variável binária com valor 1 se a pessoa de referência é mulher e valor zero se é homem.

6) Uma variável binária que assume valor 1 se a pessoa de referência tem mais de 55 anos de idade e assume valor zero em caso contrário.

7) Duas variáveis binárias para diferenciar 3 categorias de cor da pessoa de referência do domicílio: branco (adotado como base), preto ou pardo e amarelo (os indígenas foram excluídos da amostra).

8) Razão entre o número total de pessoas e o número de cômodos do domićlíio.

9) Cinco variáveis binárias para distinguir 6 categorias de tamanho do domicílio (número de pessoas do domicílio, exclusive pensionistas, empregados domésticos e parentes de empregados domésticos): 1, 2, 3, 4 (base), 5 ou 6 e 7 ou mais.

10) Uma variável binária igual a 1 quando o domicílio tem luz elétrica.

11) Variável binária para a existência de água canalizada em pelo menos um cômodo do domicílio.

12) Uma variável binária igual a 1 quando o banheiro ou sanitário do domićlio está ligado à rede coletora de esgoto ou pluvial ou a uma fossa séptica.

13) Cinco variáveis binárias para distinguir 6 regiões: Norte, Nordeste (base), MG+ES+RJ, SP, Sul e CentroOeste.

14) Uma variável binária igual a 1 quando o domićlio está em área rural e igual a zero quando está em área urbana.

15) Variável binária igual a 1 se a pessoa de referência do domicílio tem atividade principal no setor agrícola e é igual a zero em qualquer outra situação. 
16) Seis variáveis binárias para distinguir 7 posições na ocupação: empregado não-doméstico com carteira de trabalho assinada (base), militar ou funcionário público estatutário, empregado não-doméstico sem carteira assinada, empregado doméstico, trabalhador por conta própria, empregador e outras condições.

17) Fração do rendimento domiciliar formada por aposentadorias e pensões pagas pelo poder público. Inclui pagamentos do INSS e aposentadorias e pensões de funcionários públicos estatutários.

18) Variável binária que assume valor 1 se um dos membros do domicílio tem menos de 18 anos de idade. 


\section{APÊNDICE B. Um modelo de lógite para insegurança alimentar em 2009: resultados considerando ou não a estrutura complexa da amostra da PNAD}

A Tabela 10 mostra os resultados obtidos estimando um modelo de lógite para a Insegurança Alimentar Moderada ou Grave (IAMG) utilizando apenas os dados de 2009. Nesse caso, como o coeficiente do cubo do logaritmo da RDPC não se mostrou significativo, optamos por eliminar esse termo do modelo. O máximo do arco de parábola estimado ocorre com RDPC igual a $\mathrm{R} \$ 14,3$, mostrando que a função é decrescente no intervalo relevante.

Os resultados são perfeitamente coerentes com os apresentados na Tabela 8.

A Tabela 10 permite comparar a probabilidade caudal dos testes de hipóteses (usualmente denominada " $p$ value" ou "valor $p$ ") considerando ou não a estrutura complexa da amostra da PNAD [23].

Nos últimos anos, graças à disponibilidade de programas para computador que permitem levar em consideração essa estrutura complexa, o tema ganhou atenção. Mas não há programas prontos que levem em consideração a estrutura da amostra resultante do "empilhamento" dos dados das amostras de 2004 e 2009 . Assim, as estimativas foram obtidas fazendo a ponderação pelo fator de expansão de cada observação, mas sem levar em conta a estrutura complexa da amostra.

Utilizando apenas os dados de um ano, é possível fazer a análise estatística levando em consideração a estrutura complexa da amostra ${ }^{9}$. É importante ter em mente que o fato de levar em consideração a estrutura complexa da amostra não afeta as estimativas dos parâmetros, mas apenas as estimativas das variâncias e, consequentemente, as estimativas dos desvios padrões, os valores dos testes estatísticos e as respectivas probabilidades caudais.

Comparando as duas últimas colunas da Tabela 10, verifica-se que há casos de aumento substancial da probabilidade caudal quando se leva em consideração a estrutura complexa da amostra, como para o efeito de luz elétrica ou o efeito de o domicílio estar na Região Norte, em comparação com ser do Nordeste. Mas em geral não há alteração qualitativa no resultado do teste de hipótese. Se, por exemplo, for adotado um nível de significância de 1\%, o único coeficiente de variável explanatória para o qual haveria mudança de resultado "significativo" para "não significativo" seria o referente à Região Norte. É claro que, sempre que possível devemos fazer a análise estatística mais apropriada, levando em consideração a estrutura da amostra. Mas é importante saber que basta levar em consideração o fator de expansão para obter as estimativas corretas dos parâmetros e que os resultados qualitativos (no sentido de considerar ou não a estimativa obtida significativamente diferente de zero ou não) em geral não são alterados.

\footnotetext{
${ }_{9}^{9}$ No caso do $S A S$, ao estimar um modelo de lógite é essencial normalizar os pesos, de maneira que sua soma seja igual ao número de observações. Para levar em consideração a estrutura da amostra usa-se o procedimento SURVEYLOGIT em lugar do LOGIT, com os comandos "STRATA V4617” e "CLUSTER V4618”, onde V4617 e V4618 são variáveis disponíveis nos microdados da PNAD de 2009.
} 
Tabela 10. Modelo de lógite para a probabilidade de um domićlio ter Insegurança Alimentar Moderada ou Grave. Brasil, 2009

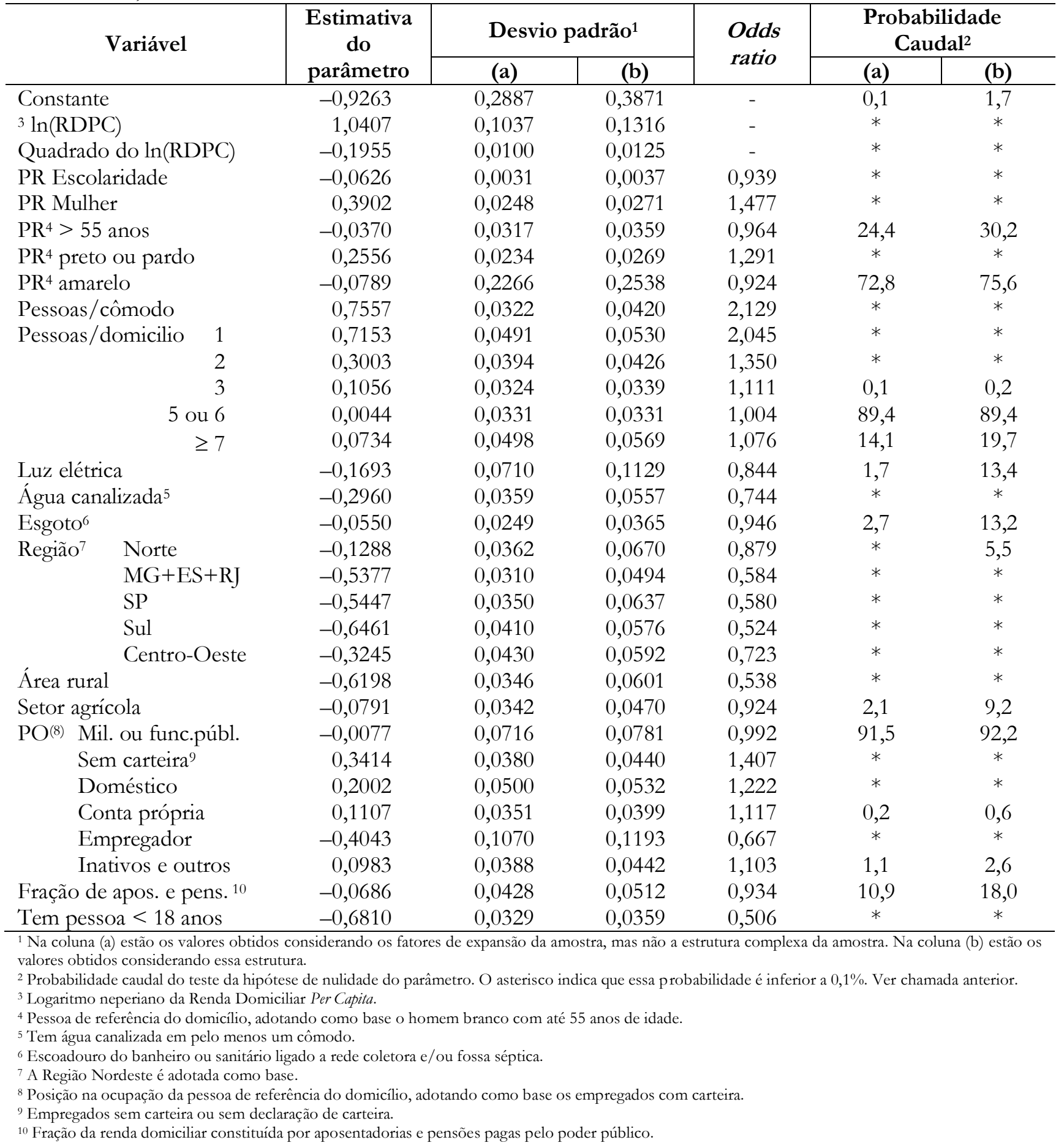

